\title{
Comparison of measurements of the outer scale of turbulence by three different techniques
}

\author{
Aziz Ziad, Matthias Schöck, Gary A. Chanan, Mitchell Troy, Richard Dekany, \\ Benjamin F. Lane, Julien Borgnino, and François Martin
}

\begin{abstract}
We have made simultaneous and nearly simultaneous measurements of $\mathscr{L}_{0}$, the outer scale of turbulence, at the Palomar Observatory by using three techniques: angle-of-arrival covariance measurements with the Generalized Seeing Monitor (GSM), differential-image-motion measurements with the adaptiveoptics system on the Hale 5-m telescope, and fringe speed measurements with the Palomar Testbed Interferometer (PTI). The three techniques give consistent results, an outer scale of approximately 10-20 m, despite the fact that the spatial scales of the three instruments vary from $1 \mathrm{~m}$ for the GSM to $100 \mathrm{~m}$ for the PTI. (C) 2004 Optical Society of America
\end{abstract}

OCIS codes: $\quad 010.1080,010.1290,010.1330,120.3180$.

\section{Introduction}

In the context of astronomical observations, the outer scale of atmospheric turbulence can be thought of as the (horizontal) distance over which the phase structure function begins to saturate and to fall significantly below the well-known $5 / 3$ power law associated with Kolmogorov turbulence. The actual size of the outer scale has long been controversial, with measured values ranging from less than $10 \mathrm{~m}$ (Refs. 1-3) to more than $2 \mathrm{~km} .{ }^{4}$ What is not controversial is the conclusion that when the diameter of the telescope approaches or exceeds the size of the outer scale, the optical consequences of atmospheric turbulence are changed dramatically from their traditional Kolmogorov behavior. In particular, power in the lowest Zernike aberration $\operatorname{modes}^{5-7}$ (e.g., tip and tilt) and the overall stroke required for an

A. Ziad (aziz.ZIAD@unice.fr), J. Borgnino, and F. Martin are with Unité Mixte de Recherche 6525 Astrophysique, Université de Nice-Sophia Antipolis, Parc Valrose, 06108 Nice Cedex 2, France. M. Schöck and G. A. Chanan are with the Department of Physics and Astronomy, University of California, Irvine, Irvine, California 92697. M. Troy is with the Jet Propulsion Laboratory, Pasadena, California 91109. R. Dekany is with the California Institute of Technology, Pasadena, California 91125. B. F. Lane is with the Department of Geological and Planetary Sciences, 150-21, California Institute of Technology, Pasadena, California 91125.

Received 27 June 2003; revised manuscript received 20 November 2003; accepted 2 February 2004.

0003-6935/04/112316-09\$15.00/0

(C) 2004 Optical Society of America adaptive-optics (AO) system $^{8}$ can be much reduced. A finite outer scale has implications for interferometry as well..$^{9-11}$ With the current interest in the design of extremely large ground-based optical and infrared telescopes, ${ }^{12-18}$ reliable estimates of the true size of the outer scale have assumed considerable importance.

In this paper we describe simultaneous measurements of the outer scale by three different techniques, utilizing the Generalized Seeing Monitor ${ }^{19}$ (GSM), the adaptive-optics system of the Hale 5-m telescope, and the Palomar Testbed Interferometer (PTI). The fact that these three instruments span a large range of length scales, from the 1-m GSM to the 100-m PTI, explicitly addresses the concern, often expressed about $\mathscr{L}_{0}$ investigations, that one may in fact be measuring not $\mathscr{L}_{0}$ but rather the scale of the apparatus itself.

In Section 2 we briefly describe each instrument and how the outer scale is extracted from its particular data set. To place the $\mathscr{L}_{0}$ results in the context of the overall turbulence conditions that are present during the observations we utilize the GSM (the most versatile of the three instruments in this regard) to extract other seeing parameters, including atmospheric coherence diameter $r_{0}$, isoplanatic angle $\theta_{0}$, and angle of arrival coherence time $\tau_{0, A A}$, in addition to $\mathscr{L}_{0}$; these results are presented in Subsection 3.A below. The GSM results are compared with those for the Palomar AO system and the PTI in Subsections 3.B and 3.C, respectively. Our conclusions are given in Section 4. 


\section{Instruments of the Palomar Observatory Site-Testing Campaign}

\section{A. Generalized Seeing Monitor}

The GSM instrument evaluates the optical parameters of the wave front perturbed by turbulence by measuring angle-of-arrival (AA) fluctuations. Its basic mode of operation is the same as that of a Shack-Hartmann sensor; i.e., the AA is measured at different points of the wave front. Computing the AA's spatiotemporal correlations leads to estimates of $r_{0}$, seeing FWHM, isoplanatic angle $\theta_{0}$, and AA coherence time $\tau_{0, A A}$ as well as outer-scale $\mathscr{L}_{0} \cdot{ }^{19}$

The instrument consists of four 10-cm telescopes on equatorial mounts equipped with detection modules to measure the AA fluctuations and interfaced to a computer that manages the four modules simultaneously. The telescopes, pointing at the same star, measure the AA fluctuations by flux modulation, which is produced by displacement of the stellar image over a Ronchi grating. Two telescopes are installed on a common mount on a central pier working as a differential image motion (DIM) monitor with a $25-\mathrm{cm}$ baseline. Two other telescopes each have independent mounts on separate piers, located $0.8 \mathrm{~m}$ to the south and $1 \mathrm{~m}$ to the east of the central pier, forming an L-shaped configuration. This geometry was chosen to increase the AA's covariance sensitivity to the outer scale. In the observations described here the telescopes were located $2 \mathrm{~m}$ above the ground.

The AA fluctuations are measured with 5-ms time resolution and 2-min acquisition time. Data are processed immediately after each acquisition, permitting quasi-real-time monitoring of the turbulence parameters. The data acquisition is typically repeated every $4 \mathrm{~min}$.

Corrections for photon counting statistics and scintillation noise are made before data processing. ${ }^{19}$ We make a correction for finite exposure time also by computing the AA variance (or covariance) for 5 and $10 \mathrm{~ms}$ and by extrapolating linearly to zero exposure time. Finally, the statistical errors of the computed variances and covariances are estimated and propagated to give the errors in the derived quantities.

The differential variance of the AA on the $25-\mathrm{cm}$ baseline is used to compute $r_{0}$ and the seeing FWHM as with a normal DIM instrument. Scintillation in$\operatorname{dex} \sigma_{I}^{2}$ is computed and used to estimate the isoplanatic angle. ${ }^{19}$ To determine $\mathscr{L}_{0}$ we compute the AA covariance for each baseline (six baselines with four GSM modules) and normalize it by the differential variance on the $25-\mathrm{cm}$ baseline. These covariances are then compared with von Kármán theoretical normalized covariances, ${ }^{20}$ and the appropriate $\mathscr{L}_{0}$ is found for each baseline. The final value of $\mathscr{L}_{0}$ is taken as the median of the six individual $\mathscr{L}_{0}$ values, and its error is estimated as described by Ziad et al. ${ }^{19}$

\section{B. Palomar Adaptive-Optics System}

The Palomar Adaptive-Optics system²1,22 (PALAO) is a facility-class AO system, developed jointly by the
Jet Propulsion Laboratory and Caltech, and has been in use at the $f / 15.7$ Cassegrain focus of the 5-m telescope since March of 1999. At the time of these experiments, the PALAO routinely achieved 50\% $K$-band Strehl ratios on natural guide stars brighter than 10th magnitude in the presence of 1-arc sec seeing (500-nm wavelength) and wind velocities of $5-10 \mathrm{~m} / \mathrm{s}$. The PALAO is based on a $16 \times 16$ element Shack-Hartmann wave-front sensor and a Xinetics, Inc., deformable mirror with 241 actively controlled actuators. During September 2001 the system was operated with $4 \times 4$ pixels per subaperture for calculation of Shack-Hartmann centroids and the wave-front sensor frame rate was $500 \mathrm{~Hz}$, with 6-electron read noise at 2.5-Mpixels/s readout at each of the four ports. The infrared science camera used in conjunction with the PALAO is the Palomar High-Angular-Resolution Observer (PHARO) built by Cornell University and based on a $1024 \times 1024$ Rockwell Hawaii-2 HgCdTe array.

Values for atmospheric turbulence parameters can be obtained from the PALAO telemetry data. The AO system is capable of recording continuous telemetry at acquisition rates of $100 \mathrm{~Hz}$. Because the AO system was operating at frame rates of $500 \mathrm{~Hz}$ at the time of our observations, every fifth frame was captured. All data analyzed for this paper were taken either with the deformable mirror loop open and the tip-tilt (TT) loop closed, hereafter referred to as the TT-loop-closed mode, or in closed-loop mode, that is, with both the deformable mirror and the TT loops closed.

In TT-loop-closed operation the telemetry data of interest are the centroids measured by the AO wavefront sensor. In this case there are two methods by which one can obtain atmospheric parameters: analysis of DIM among various wave-front sensor subapertures and analysis of the Zernike decomposition of the reconstructed wave-front phase. Both methods have been described in depth elsewhere. ${ }^{23}$ In brief, by use of the DIM method, $\mathscr{L}_{0}$ is determined from the shape of the DIM structure function, whereas $r_{0}$ is found from the magnitude of this structure function. Similarly, the Zernike decomposition method yields $r_{0}$ when the absolute values of the Zernike mode variances are used, whereas $\mathscr{L}_{0}$ is found from the ratios of the variances of different modes. In closed-loop mode the data analyzed are the actuator positions of the deformable mirror from which the wave-front phase can be found. The subsequent analysis is then identical to the Zernike decomposition method of TT-loop-closed data.

The calculation of $r_{0}$ by these methods has been shown to be accurate, provided that the instrument has been carefully calibrated. However, previously there had been no external verification of the outerscale measurements, although the consistency of $\mathscr{L}_{0}$ values obtained from the DIM and Zernike methods has been shown. ${ }^{23}$

\section{Palomar Testbed Interferometer}

The $\mathrm{PTI}^{24}$ is a long-baseline infrared interferometer installed at the Palomar Observatory, close to the 
5 -m telescope. $\quad$ It operates in the $J(1.2 \mu \mathrm{m}), H(1.6$ $\mu \mathrm{m})$, and $K(2.2 \mu \mathrm{m})$ bands, and, with a maximum baseline of $110 \mathrm{~m}$, achieves an angular resolution of $\sim 3$ milli arc sec. It was developed by the Jet Propulsion Laboratory and the California Institute of Technology for NASA as a testbed for interferometric techniques applicable to the Keck Interferometer and the Space Interferometry Mission (SIM).

The PTI operates by combining light from two widely separated apertures with a $50 / 50$ beam splitter and measuring the phase and visibility of the resultant interference fringes. Whereas the fringe visibility can be related to the morphology of the observed source ${ }^{25}$ (by means of the van Cittert-Zernike theorem), the fringe phase measured by a singlebaseline instrument such as the PTI is too corrupted by the atmosphere to be useful for imaging purposes. In practice, therefore, the PTI system continuously (on 10-20-ms time scales) adjusts the amount of internal optical path delay to drive the fringe phase to zero (and hence maximize the measured fringe visibility). As a result, the PTI system produces a high time-resolution record of the optical path delay introduced by the atmosphere above the instrument. Typically, this atmospheric turbulence is characterized by a jitter number that corresponds to the rms difference in fringe phase between successive fringe samples.

For interferometric observations, atmospheric turbulence introduces random phase variations above each telescope. The resultant variable optical path difference between the interferometer arms produces fringe displacements across the detector, which result in blurring of the fringe pattern and therefore in a degradation of contrast. In what follows, we describe how a value for the outer scale can be extracted from the statistics of this fringe jitter.

The time structure function of the white-light phase $\phi$ for a sampling time $\tau$ is given by

$$
\sigma_{\phi, \tau}^{2}=\left\langle[\phi(x, y, t)-\phi(x, y, t+\tau)]^{2}\right\rangle,
$$

where \langle\rangle denotes the ensemble average. When $\tau$ tends to zero, Eq. (1) leads to

$$
\begin{aligned}
\sigma_{\phi, \tau}^{2} & \simeq\left\langle\left[\frac{\partial \phi(x, y, t)}{\partial t}\right]^{2}\right\rangle \tau^{2} \\
& =2\left[C_{\partial \varphi / \partial t}(0,0)-C_{\partial \varphi / \partial t}\left(b_{x}, b_{y}\right)\right] \tau^{2},
\end{aligned}
$$

where $C_{\partial \varphi / \partial t}$ denotes the covariance of wave-front phase velocity $\partial \varphi / \partial t$. The white-light fringe phase is related to the wave-front phase measured with the two telescopes separated by a baseline $\left(b_{x}, b_{y}\right)$ as $\phi(x$, $y, t)=\left[\varphi(x, y, t)-\varphi\left(x+b_{x}, y+b_{y}, t\right)\right]$.

The Taylor hypothesis supposes that atmospheric turbulence consists of a single frozen layer, transported at wind speed $v$. Thus we can describe the phase velocity by

$$
\frac{\partial \varphi(x, y, t)}{\partial t}=\frac{\partial \varphi(x, y, t)}{\partial x} \frac{\partial x}{\partial t}+\frac{\partial \varphi(x, y, t)}{\partial y} \frac{\partial y}{\partial t},
$$

where

$$
\frac{\partial x}{\partial t}=v \cos \theta, \quad \frac{\partial y}{\partial t}=v \sin \theta,
$$

and $\theta$ is the angle between the $x$ axis and the line joining the origin with point $(x, y)$ on the wave front.

Using the AA definitions in the $x$ and $y$ directions yields

$$
\alpha=-\frac{\lambda}{2 \pi} \frac{\partial \varphi(x, y)}{\partial x}, \quad \beta=-\frac{\lambda}{2 \pi} \frac{\partial \varphi(x, y)}{\partial y}
$$

for wavelength $\lambda$; taking the $x$ axis to be in the same direction as the baseline, we find that

$$
\begin{aligned}
C_{\partial \varphi / \partial t}\left(b_{x}, 0\right)= & \left(\frac{2 \pi v}{\lambda}\right)^{2}\left[C_{\alpha}\left(b_{x}, 0\right) \cos ^{2} \theta\right. \\
& \left.+C_{\beta}\left(b_{x}, 0\right) \sin ^{2} \theta\right] .
\end{aligned}
$$

The AA covariance has no analytical expression but can be deduced from AA power spectrum $W_{\alpha / \beta}\left(f_{x}\right.$, $\left.f_{y}\right)=\lambda^{2} f_{x / y}{ }^{2} W_{\varphi}\left(f_{x}, f_{y}\right)$ by use of the inverse Fourier transform ${ }^{20}$ (Wiener-Khinchin theorem). $W_{\varphi}\left(f_{x}, f_{y}\right)$ is the wave-front phase power spectrum and $f_{x}$ and $f_{y}$ indicate spatial frequencies in the $x$ and $y$ directions, respectively:

$$
\begin{aligned}
C_{\alpha / \beta}\left(b_{x}, 0\right)= & \lambda^{2} \iint f_{x / y}{ }^{2} W_{\varphi}\left(f_{x}, f_{y}\right) \exp \left(-2 i \pi b_{x} f_{x}\right) \\
& \times\left[\frac{2 J_{1}(\pi D f)}{\pi D f}\right]^{2} \mathrm{~d} f_{x} \mathrm{~d} f_{y},
\end{aligned}
$$

where $J_{1}$ is the first-order Bessel function and $D$ is the telescope diameter.

We can now relate the AA covariance to outer scale $\mathscr{L}_{0}$ by assuming an outer-scale dependence for phase power spectral density $W_{\varphi}$. We adopt the von Kármán model for the latter:

$$
W_{\varphi}\left(f_{x}, f_{y}\right)=0.0229 r_{0}{ }^{-5 / 3}\left(f_{x}^{2}+f_{y}^{2}+\mathscr{L}_{0}{ }^{-2}\right)^{-11 / 6},
$$

where $r_{0}$ is the Fried parameter and the numerical factor and the power law are chosen such that the right-hand side of the equation approaches the Kolmogorov spectrum as $\mathscr{L}_{0}$ becomes large. Other models for the dependence of $W_{\varphi}$ on $\mathscr{L}_{0}$ may be chosen ${ }^{26}$ but are unlikely to change the inferred value of $\mathscr{L}_{0}$ by a large factor. Equations (6) and (7) now provide the desired dependence of the AA covariance on the outer scale, and one can infer an estimate for the latter by fitting the data to the expression for the former. In Subsection 3.C a comparison of the PTI fringe jitter data and this model based on the GSM $r_{0}$ results is used for the outer-scale $\mathscr{L}_{0}$ estimation.

\section{Results}

\section{A. Generalized Seeing Monitor}

A summary of the results for the various seeing parameters obtained with the GSM from 5 to 15 September 2001 is presented in Fig. 1. Each 


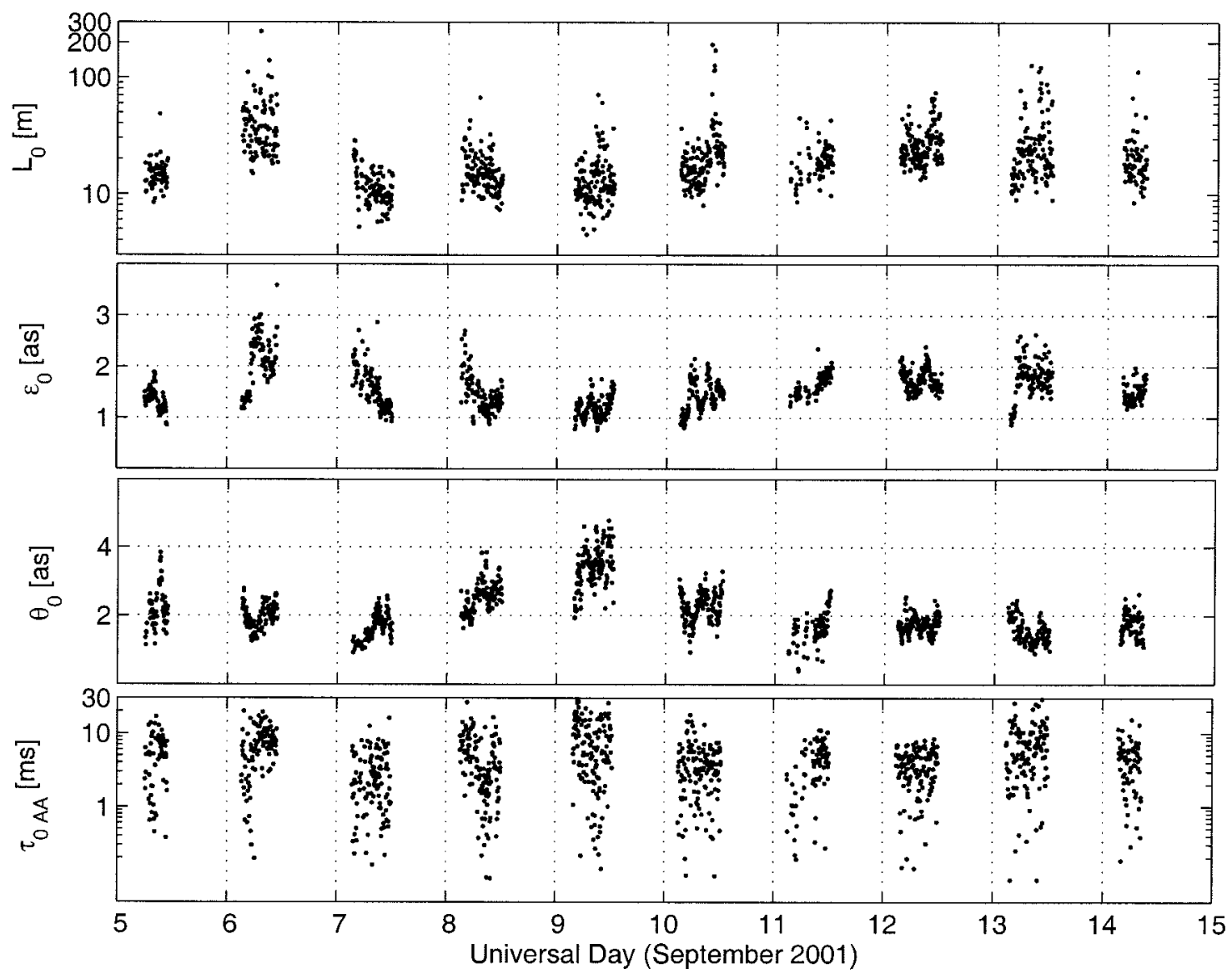

Fig. 1. Atmospheric parameters measured by the GSM at the Palomar Observatory: $\mathscr{L}_{0}$ is the outer scale of turbulence, $\epsilon_{0}$ is the seeing FWHM (as inferred from atmospheric coherence diameter $r_{0}$ ), $\theta_{0}$ is the isoplanatic angle, and $\tau_{0 A A}$ is the angle-of-arrival atmospheric coherence time.

measurement corresponds to a 2-min acquisition time, and a new data set is taken every $4 \mathrm{~min}$. All atmospheric parameters are scaled to the wavelength $\lambda=0.5 \mu \mathrm{m}$. Note the large number of measurements during this campaign compared with campaigns conducted at other sites. ${ }^{19}$ Log-normal distributions are generally useful for the characterization of parameters encountered in atmospheric turbulence, ${ }^{27-29}$ and in fact the various parameters measured here are all reasonably well fitted by such distributions, as shown in Fig. 2.

Typical values for $\mathscr{L}_{0}$ are of the order of $10-20 \mathrm{~m}$ (Fig. 1), somewhat smaller than the GSM results from other sites. As with several other sites, some bursts of high $\mathscr{L}_{0}$ are seen, which typically last for a few minutes; these bursts may correspond to newly generated turbulence. In general, the high variability ${ }^{19}$ of $\mathscr{L}_{0}$ may mean that for the optimization of $\mathrm{AO}$ systems, including PSF modeling, ${ }^{30}$ and for longbaseline interferometry it may be desirable to monitor the outer scale.

We also employ here a new method for estimating the AA coherence time $\tau_{0, A A}$ in real time. ${ }^{31}$ This method consists of processing the AA temporal structure function

$$
\sigma_{\alpha}^{2}(r, \tau)=\left\langle[\alpha(r, t)-\alpha(r, t+\tau)]^{2}\right\rangle,
$$

where $\alpha$ indicates the AA measured with the GSM over a $10-\mathrm{cm}$ telescope diameter. This AA is measured with a GSM module at spatial position $r$ and at regular time intervals $\tau$ (multiples of $5 \mathrm{~ms}$ ). This temporal structure function $\sigma_{\alpha}{ }^{2}(r, \tau)$ saturates for large values of $\tau$; the point at which the structure function reaches $1 / e$ of this maximum corresponds to the AA coherence time. To distinguish this parameter from the $\tau_{0}$ that is deduced from the wave-front phase structure function ${ }^{31}$ we denote the GSM coherence time $\tau_{0, A A}$.

\section{B. Comparison of Generalized Seeing Monitor and Palomar Adaptive-Optics System Data}

Measurements with the PALAO were taken during the nights of 7-9 September 2001, a subset of the GSM nights of 5-15 September. A total of approximately 460,000 frames (77 $\mathrm{min})$ of TT-loop-closed data and 195,000 frames (33 min) of closed-loop data were taken throughout the three nights.

A comparison of $r_{0}$ measurements from the PALAO and the GSM is shown in Fig. 3. During the periods of simultaneous measurements, GSM data were taken every $3 \mathrm{~min}$, with acquisition times of $2 \mathrm{~min}$ for each data set. PALAO data were taken as continuous time series of 5-15-min length. For comparison 

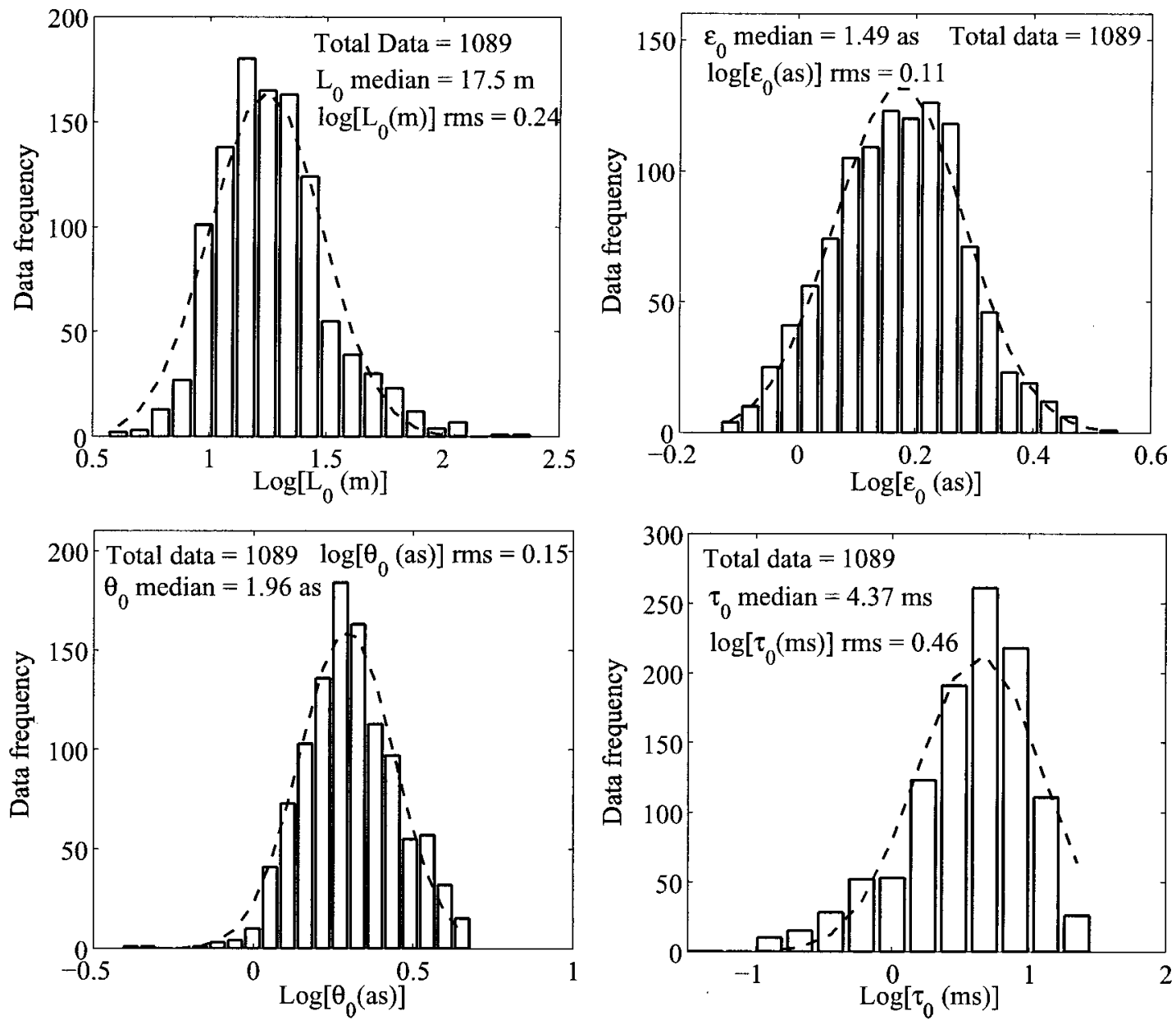

Fig. 2. Distributions of the atmospheric optical parameters obtained with the GSM at the Palomar Observatory in September 2001. Dashed curves, best-fit log-normal distributions.

purposes, each GSM data set was cut into two 1-min pieces. The PALAO data were divided into 1-min sets that were simultaneous with the GSM data sets. As described in Subsection 2.B, results from both the DIM and the Zernike methods are available for TTloop-closed data, whereas only Zernike method results can be obtained for closed-loop data. Thus the TT-closed data in Fig. 3 are those that display two PALAO $r_{0}$ values, whereas the closed-loop data show only results from the Zernike method.

As can be seen from Fig. 3, the PALAO DIM and Zernike $r_{0}$ results are in good agreement, although Zernike results display significantly higher scatter. This is a general characteristic of PALAO results and may be caused by slowly evolving dome turbulence, which, even on time scales of tens of seconds, does not produce statistically representative Zernike mode variances. (The idea that this scatter is characteristic of the Palomar telescope and is not merely an artifact of the method is supported by the fact that corresponding data from the Keck telescopes, which have much better-ventilated domes, do not show similar scatter. ${ }^{23}$ ) If time averages of several minutes are considered, the scatter of the Zernike results de- creases and excellent agreement between the DIM and Zernike methods is achieved.

Thus the DIM method results are more reliable and should be compared to GSM measurements for TT-loop-closed results. The Zernike results are nevertheless also shown for TT-loop-closed data to give an impression of the accuracy of individual values produced by the Zernike method, as only this method is available for closed-loop data.

A direct comparison of the $r_{0}$ values measured by the GSM and the PALAO is not possible because of the different locations of the two instruments. The GSM was set up between the arms of the PTI, surrounded by trees and lower than some of the surrounding terrain; the 5-m telescope is located above all surrounding relief and approximately $200 \mathrm{~m}$ southwest of and $30 \mathrm{~m}$ above the GSM location. Thus we expected the GSM to see much stronger effects of the ground layer than the PALAO system. It is nevertheless worth noting that there is a good qualitative agreement between the GSM and PALAO results most of the time, with the expected quantitative difference that the PALAO system experienced less turbulence overall than the GSM. The only 

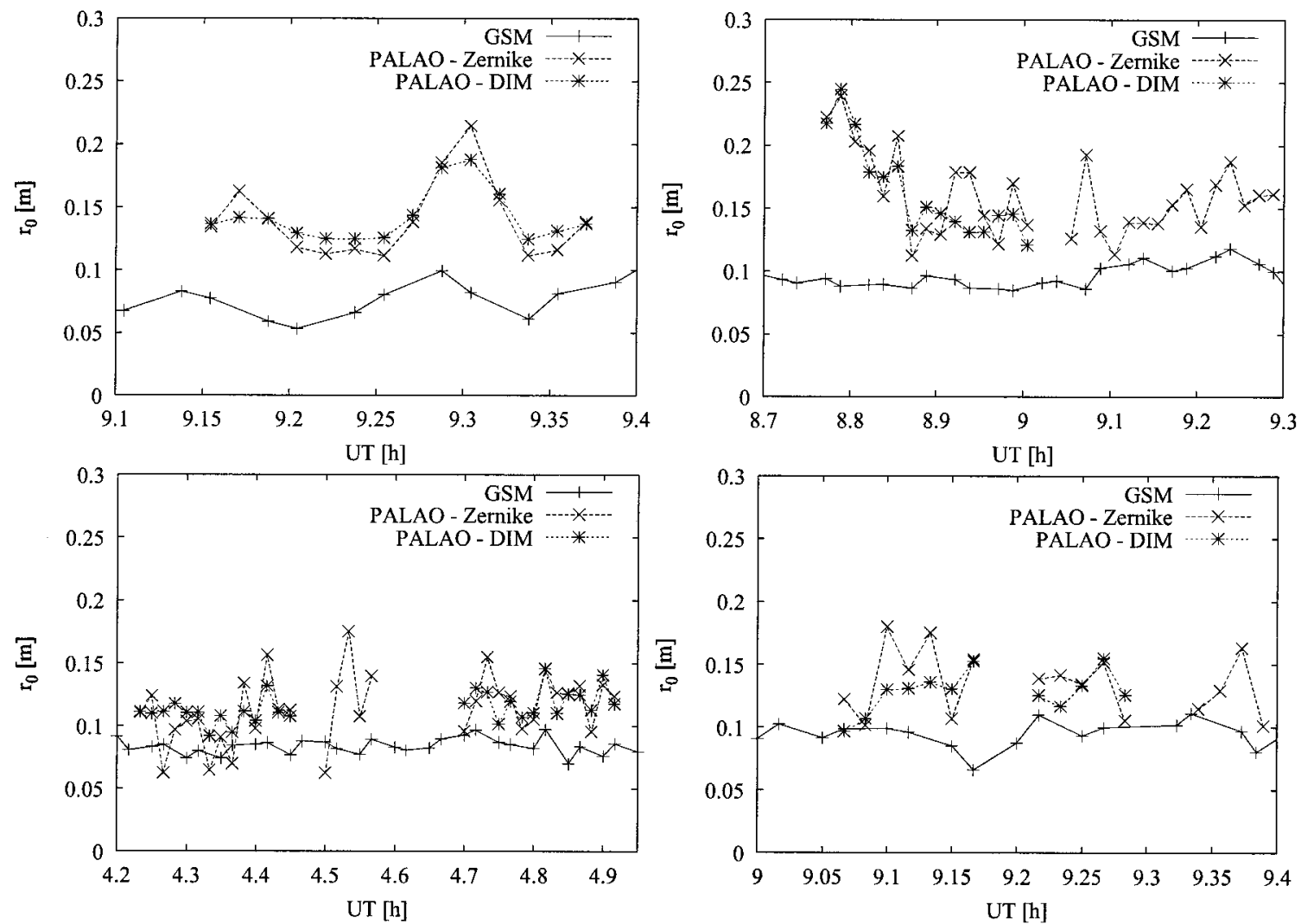

Fig. 3. Comparison of atmospheric coherence length $r_{0}$ measurements obtained with the GSM and from PALAO telemetry data during the nights of 7, 8, and 9 September 2001 (respectively, top left, top right, and bottom) at the Palomar Observatory. Differences are due to the different locations of the instruments and display the expected behavior, as explained in the text.

time when there was a significant qualitative difference between the two instruments was at the beginning of the measurements on 8 September. In this case the seeing was likely dominated by ground-layer turbulence, which, before approximately 8.9 h UT, appeared to be located mainly below the primary mirror of the 5-m telescope.

The $\mathscr{L}_{0}$ measurements obtained from the PALAO are shown in Fig. 4. Because the $\mathscr{L}_{0}$ determination from AO data is much noisier than that of $r_{0}, 2-\mathrm{min}$ simultaneous time averages are used for the comparison of PALAO and GSM results. As $\mathscr{L}_{0}$ is a largerscale quantity than $r_{0}$, a quantitative comparison of the GSM and PALAO results is more meaningful for $\mathscr{L}_{0}$ than for $r_{0}$. Given that it is difficult in general to measure $\mathscr{L}_{0}$ and that turbulence characteristics change only slowly with varying $\mathscr{L}_{0}$, the agreement between the values obtained from the two instruments is very good. The occasional spikes in the $\mathscr{L}_{0}$ values of the PALAO results have also been seen in GSM data (see Fig. 1) and are believed to be caused by real local and short-term changes of atmospheric turbulence.

\section{Comparison of Generalized Seeing Monitor and} Palomar Testbed Interferometer Data

Figure 5 shows a typical comparison between fringe speed measured with the PTI and modeled with the
GSM data $\left(r_{0}, \mathscr{L}_{0}\right)$, as discussed in Subsection 2.C. As the wind speed profiles were not measured during the observations, the GSM data in Fig. 5 were deduced by use of $v=10 \mathrm{~m} / \mathrm{s}$ in Eqs. (4). This wind speed value was chosen for visual agreement between the GSM and PTI data. Thus the residual difference between the GSM and the PTI data in Fig. 5 is due to the variation of the wind speed during the observations. We remark that for the PTI configuration (110-m baseline oriented $20^{\circ}$ East of North), wind direction $\theta$ has no significant effect on the results.

By combining these PTI data and the GSM $r_{0}$ results it is also possible to deduce estimates of outer scale $\mathscr{L}_{0}$. That is, each fringe speed measured with the PTI (Fig. 5) can be fitted with the theoretical form in relation (2) by use of the von Kármán model [Eq. (7)] and the $r_{0}$ value measured with the GSM at the same time. Thus the relative difference $R D$ between the PTI data and the theoretical model $(R D=$ $\left.\left|\sigma_{\phi, \tau, P T I}-\sigma_{\phi, \tau, \text { model }}\right| / \sigma_{\phi, \tau, P T I}\right)$ depends only on outer scale $\mathscr{L}_{0}$ and on wind speed $v$. The pair $\left(\mathscr{L}_{0}, v\right)$ that gives the minimum of $R D$ should constitute the optimal solution of this fitting. But, knowing that the error in $r_{0}$ measured with the GSM is $\sim 1 \%, 19$ we keep the same value as the upper limit of relative difference $R D$ between the PTI data and theory. Therefore different solutions of $\left(\mathscr{L}_{0}, v\right)$ are obtained for $R D \leq 1 \%$. The value of $\mathscr{L}_{0}$ that is ultimately 

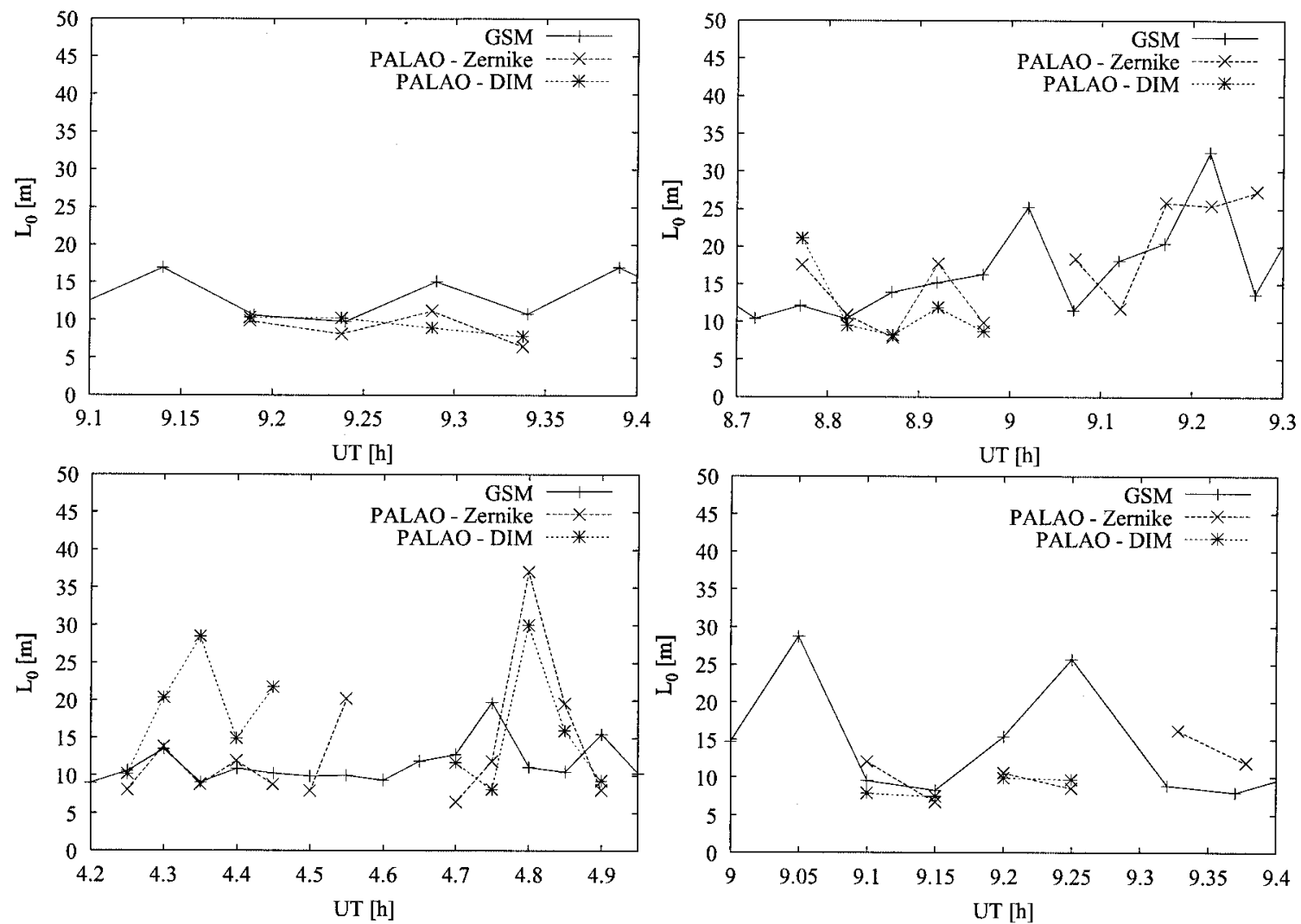

Fig. 4. Comparison of outer-scale of turbulence $\left(\mathscr{L}_{0}\right)$ measurements obtained with the GSM and from PALAO telemetry data during the nights of 7, 8, and 9 September 2001 (respectively, top left, top right, and bottom) at the Palomar Observatory. These results are to our knowledge the first independent verification of the validity of our method to determine $\mathscr{L}_{0}$ from AO telemetry data.

adopted is taken as the median of the different solutions. The mean difference between the $\mathscr{L}_{0}$ median value and the other solutions is as much as $54 \%$. Typical PTI outer-scale values are shown in Fig. 6 for comparison with the GSM results. The mean value

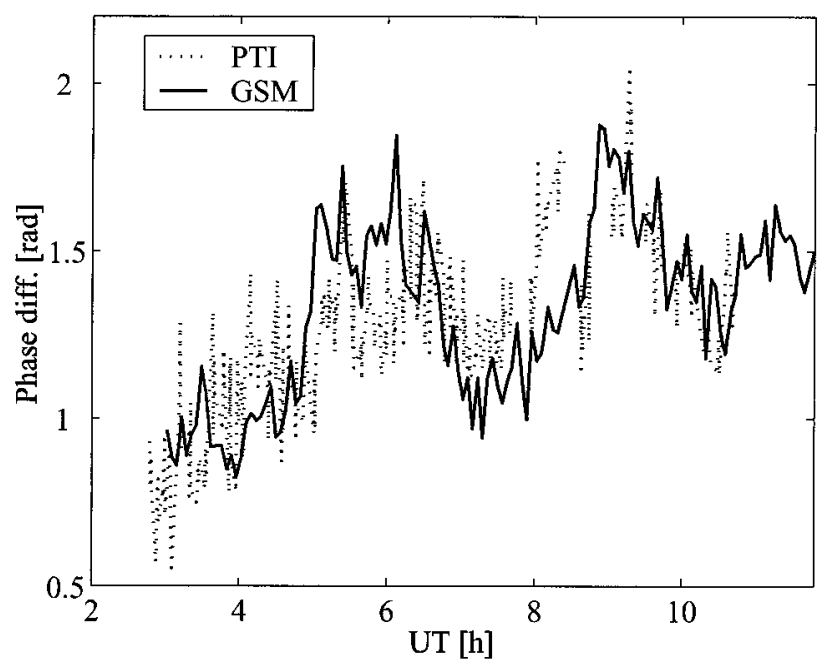

Fig. 5. Typical results for temporal differential variance (fringe speed multiplied by sampling time) measured with the PTI interferometer and modeled from the GSM data. These data were obtained during the night of 9 September 2001 at the Palomar Observatory. of the corresponding wind speed $v$ for the data in this figure was $13.3 \mathrm{~m} / \mathrm{s}$. These $\mathscr{L}_{0}$ results are in good agreement with those obtained by Linfield et al..$^{32}$ with the same instrument by use of the phase struc-

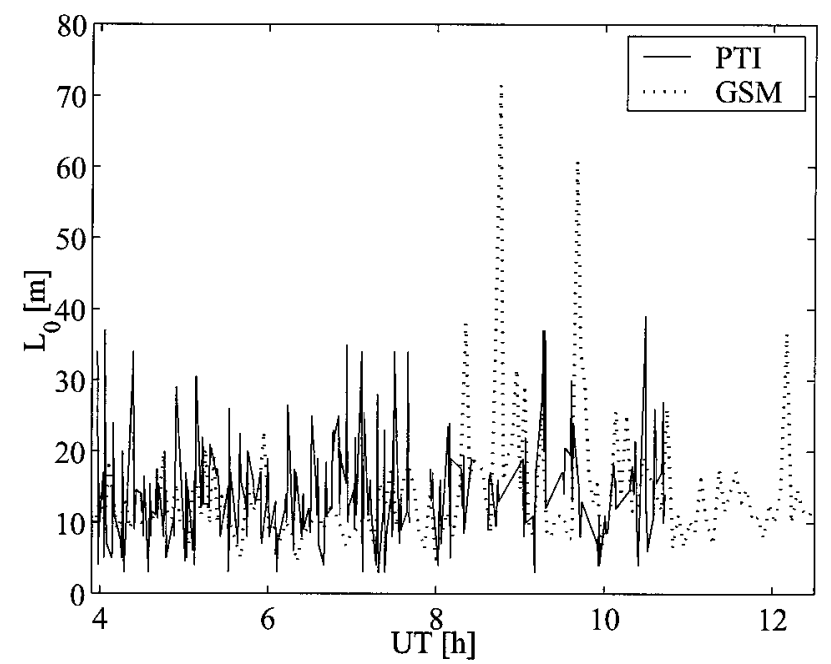

Fig. 6. Typical results for outer-scale of turbulence $\mathscr{L}_{0}$ as measured by the GSM and by the PTI interferometer. We deduced the $\mathscr{L}_{0}$ values obtained with the PTI by fitting the measured fringe speed with the theoretical expression given in Subsection 2.C. These data were obtained during the night of 9 September 2001 at the Palomar Observatory. 
ture function saturation. Despite the baseline difference between the GSM $(\sim 1 \mathrm{~m})$ and the PTI $(\sim 110$ $\mathrm{m})$ there is good agreement between the values obtained from the two instruments.

\section{Conclusions}

We measured the outer scale $\mathscr{L}_{0}$ simultaneously, using three different instruments. The results lie mainly in the range $10-20 \mathrm{~m}$ and are consistent among the various methods, despite the fact that the spatial scales of the respective instruments span 2 orders of magnitude. As usual with atmospheric turbulence data, results from a single site are not necessarily of universal applicability, but we note that our results here for $\mathscr{L}_{0}$ are generally consistent with, although somewhat smaller than, what has been found at other sites. ${ }^{19}$ If these results are representative of other sites, then it will be essential to take outer-scale effects into account when one is predicting the optical performance of extremely large optical and infrared telescopes, i.e., those with diameters of $30 \mathrm{~m}$ or more.

This research has been supported in part by the National Science Foundation Science and Technology Center for Adaptive Optics, which is managed by the University of California, Santa Cruz, under cooperative agreement AST-9876783. We are grateful to R. Thicksten and the staff of the Palomar Observatory for the excellent support that we enjoyed during the course of these observations.

\section{References}

1. C. E. Coulman, J. Vernin, Y. Coqueugniot, and J. L. Caccia, "Outer scale of turbulence appropriate to modeling refractiveindex structure profiles," Appl. Opt. 27, 155-160 (1988).

2. N. S. Nightingale and D. F. Buscher, "Interferometric seeing measurements at the La Palma Observatory," Mon. Not. R. Astron. Soc. 251, 155-166 (1991).

3. M. Bester, W. C. Danchi, C. G. Degiacomi, L. J. Greenhill, and C. H. Townes, "Atmospheric fluctuations-structure functions and projected performance of future instruments," Astrophys. J. 392, 357-374 (1992).

4. M. M. Colavita, M. Shao, and D. H. Staelin, "Atmospheric phase measurements with the Mark III stellar interferometer," Appl. Opt. 26, 4106-4112 (1987).

5. D. M. Winker, "Effect of a finite outer scale on the Zernike decomposition of atmospheric optical turbulence," J. Opt. Soc. Am. A 8, 1568-1573 (1991).

6. V. Voitsekhovich and S. Cuevas, "Adaptive optics and the outer scale of turbulence," J. Opt. Soc. Am. 12, 2523-2531 (1995).

7. R. Conan, "Modélisation des effets de l'échelle externe de cohérence spatiale du front d'onde pour l'observation à haute résolution angulaire en astronomie," Ph.D. dissertation (Nice University, Nice, France, 2000).

8. M. Le Louarn, N. Hubin, M. Sarazin, and A. Tokovinin, "New challenges for adaptive optics: extremely large telescopes," Mon. Not. R. Astron. Soc. 317, 535-544 (2000).

9. F. Roddier, "The effects of atmospheric turbulence in optical astronomy," Prog. Opt. 19, 281-376 (1981).

10. A. Ziad, J. Borgnino, A. Agabi, and F. Martin, "Optimized spectral bandwidth in high angular resolution imaging. Effect of a finite spatial-coherence outer scale," Exp. Astron. 5, 247-268 (1994).

11. J. M. Mariotti, "Adaptive optics for long baseline optical inter- ferometry," in Adaptive Optics for Astronomy, D. M. Alloin and J.-M. Mariotti, eds. (Kluwer Academic, Dordrecht, The Netherlands, 1994), pp. 309-320.

12. J. E. Nelson, "Progress on the California Extremely Large Telescope (CELT)," in Future Giant Telescopes, J. R. P. Angel and R. Gilmozzi, eds., Proc. SPIE 4840, 47-59 (2002).

13. T. Anderson, A. L. Ardeberg, J. Beckers, A. Goncharov, M. Owner-Petersen, H. Riewaldt, R. Snel, and D. Walker, "The Euro-50 extremely large telescope," in Future Giant Telescopes, J. R. P. Angel and R. Gilmozzi, eds., Proc. SPIE 4840, 214-225 (2002)

14. J. R. P. Angel, J. H. Burge, J. L. Codona, W. B. Davison, and B. Martin, "20- and 30-m telescope designs with potential for subsequent incorporation into a track-mounted pair (20/20 or 30/30)," in Future Giant Telescopes, J. R. P. Angel and R. Gilmozzi, eds., Proc. SPIE 4840, 183-193 (2002).

15. D. Burgarella, K. Dohlen, M. Ferrari, F. Zamkotsian, F. Hammer, F. Sayed, and R. Rigaut, "Large petal telescope for the next-generation Canada-France-Hawaii Telescope," in Future Giant Telescopes, J. R. P. Angel and R. Gilmozzi, eds., Proc. SPIE 4840, 93-103 (2002).

16. P. Dierickx, J. L. Beckers, E. Brunetto, R. Conan, E. Fedrigo, R. Gilmozzi, N. N. Hubin, F. Koch, M. LeLouarn, E. Marchetti, G. J. Monnet, L. Noethe, M. Quattri, M. S. Sarazin, J. Spyromilio, and N. Yaitskova, "The eye of the beholder: designing the OWL," in Future Giant Telescopes, J. R. P. Angel and R. Gilmozzi, eds., Proc. SPIE 4840, 151-170 (2002).

17. S. C. Roberts, C. L. Morbey, D. R. Crabtree, R. Carlberg, D. Crampton, T. J. Davidge, J. T. Fitzsimmons, M. H. Gedig, D. J. Halliday, J. E. Hesser, G. Herriot, J. B. Oke, J. S. Pazder, K. Szeto, and J.-P. Veran, "Canadian very large optical telescope technology studies," in Future Giant Telescopes, J. R. P. Angel and R. Gilmozzi, eds., Proc. SPIE 4840, 104-115 (2002).

18. S. E. Strom, L. M. Stepp, and B. Gregory, "Giant Segmented Mirror Telescope: a point design based on science drivers," in Future Giant Telescopes, J. R. P. Angel and R. Gilmozzi, eds., Proc. SPIE 4840, 116-128 (2002).

19. A. Ziad, R. Conan, A. Tokovinin, F. Martin, and J. Borgnino, "From the Grating Scale Monitor to the Generalized Seeing Monitor," Appl. Opt. 39, 5415-5425 (2000).

20. R. Avila, A. Ziad, J. Borgnino, F. Martin, A. Agabi, and A. Tokovinin, "Theoretical spatiotemporal analysis of angle of arrival induced by atmospheric turbulence as observed with the grating scale monitor experiment," J. Opt. Soc. Am. A 14, 3070-3082 (1997).

21. R. G. Dekany, J. K. Wallace, G. Brack, B. R. Oppenheimer, and D. Palmer, "Initial test results from the Palomar 200-in. Adaptive Optics System," in Adaptive Optics and Applications, R. K. Tyson and R. Q. Fugate, eds., Proc. SPIE 3126, 269-276 (1997).

22. M. Troy, R. Dekany, G. Brack, B. Oppenheimer, E. Bloemhof, T. Trinh, F. Dekens, F. Shi, T. Hayward, and B. Brandl, "Palomar Adaptive Optics Project: status and performance," in Adaptive Optical Systems Technology, P. L. Wizinowich, ed., Proc. SPIE 4007, 31-40 (2000).

23. M. Schöck, D. Le Mignant, G. Chanan, P. L. Wizinowich, and M. A. Van Dam, "Atmospheric turbulence characterization with the Keck adaptive optics systems. I. Open-loop data," Appl. Opt. 42, 3705-3720 (2003).

24. M. M. Colavita, J. K. Wallace, B. E. Hines, Y. Gursel, F. Malbet, D. L. Palmer, X. P. Pan, M. Shao, J. W. Yu, A. F. Boden, P. J. Dumont, J. Gubler, C. D. Koresko, S. R. Kulkarni, B. F. Lane, D. W. Mobley, and G. T. van Belle, "The Palomar Testbed Interferometer," Astrophys. J. 510, 505-521 (1999).

25. A. F. Boden, C. D. Koresko, G. T. van Belle, M. M. Colavita, P. J. Dumont, J. Gubler, S. R. Kulkarni, B. F. Lane, D. Mobley, 
M. Shao, and J. K. Wallace, "The visual orbit of Iota Pegasi," Astrophys. J. 515, 356-364 (1999).

26. V. Voitsekhovich, "Outer scale of turbulence: comparison of different models," J. Opt. Soc. Am. A 12, 1346-1353 (1995).

27. J. M. Mariotti, "Experimental results on atmospheric turbulence obtained with an infrared speckle-interferometer," Opt. Acta 30, 831-840 (1983).

28. R. Racine, "Astronomical seeing at Mauna Kea and in particular at the CFHT," in Very Large Telescopes: Their Instrumentation and Programs, M. H. Ulrich and K. Kjär, eds., IAU Colloquium 79 (European Southern Observatory, Garching, Germany, 1984), pp. 235-243.
29. J. W. Goodman, Statistical Optics (Wiley, New York, 2000), pp. $399-400$.

30. F. Rigaut and M. Sarazin, "Seeing constraints in adaptive optics calibrations," in Astronomy with Adaptive Optics: Present Results and Future Programs, D. Bonaccini, ed., Vol. 56 of ESO Conference and Workshop Proceedings (European Southern Observatory, Garching, Germany, 1999), p. 383.

31. F. Roddier, Adaptive Optics in Astronomy (Cambridge U. Press, Cambridge, 1999).

32. R. P. Linfield, M. M. Colavita, and B. F. Lane, "Atmospheric turbulence measurements with the Palomar Testbed Interferometer," Astrophys. J. 554, 505-513 (2001). 